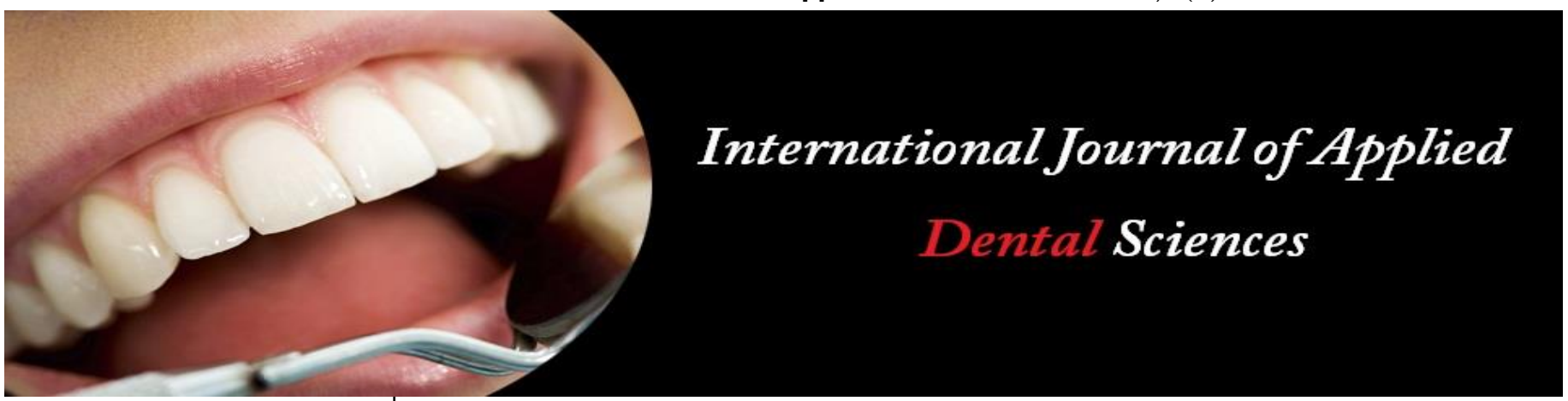

ISSN Print: 2394-7489

ISSN Online: 2394-7497

IJADS 2021; 7(1): 84-88

(C) 2021 IJADS

www.oraljournal.com

Received: 07-10-2020

Accepted: 13-11-2020

Dr. Abhishek Kumar Katiyar Associate Professor,

Prosthodontics and Crown \& Bridge and Implantology,

Chandra Dental College and

Hospital, Barabanki,

Uttar Pradesh, India

Dr. Aliza Rizvi

College of Dental Sciences and

Research, Maharishi

Markandeshwar, Ambala,

Haryana, India

\section{Dr. Milan Soni}

Non-Academic Junior Resident, Department of Dentistry, AIIMS

Bhopal, Madhya Pradesh, India

Dr. Georgee Sharun Philip Prosthodontics, Bbd Lucknow,

Chennai, Tamil Nadu, India

Dr. Siddharth David

Public Health Dentistry, BBDCODS, Lucknow, Uttar

Pradesh, India

Dr. Saundarya Priyadarshini Public Health Dentistry, BBDCODS, Lucknow, Uttar Pradesh, India

Corresponding Author: Dr. Siddharth David Public Health Dentistry, BBDCODS, Lucknow, Uttar Pradesh, India

\section{A cross-sectional prevalence study: To assess the prevalence and site distribution of oral mucosal lesions}

\author{
Dr. Abhishek Kumar Katiyar, Dr. Aliza Rizvi, Dr. Milan Soni, Dr. \\ Georgee Sharun Philip, Dr. Siddharth David and Dr. Saundarya \\ Priyadarshini
}

DOI: $\underline{\text { https://doi.org/10.22271/oral.2021.v7.i1b.1120 }}$

\section{Abstract}

Introduction: Early diagnosis is the key in the prevention of transformation of oral mucosal lesions into life threatening disease 'CANCER', hence the need of the study determines the prevalence and site distribution of Oral Mucosal Lesions in patients attending outpatient department of Shivam Dental Clinic, Lakhimpur.

Methods: A cross-sectional prevalence study was carried out to assess the prevalence and site distribution of Oral Mucosal Lesions in patients attending outpatient department of Shivam Dental Clinic, Lakhimpur. The sample size was estimated to be 150. A single examiner previously trained for the diagnosis of Oral Mucosal lesions made all examinations. The data was collected using the WHO oral health assessment form 2013. The data analysis was done using the SPSS version 20.

Result: The results stated a strong association between age, chewing tobacco smoking and oral lesions. Conclusion: The present study concludes a positive relation between intake of tobacco in any form with oral mucosal lesions hence an additional effort to educate the masses about the hazardous effects of tobacco should be a priority for both governmental and non-governmental agencies.

Keywords: Oral mucosal lesions, tobacco, oral cancer, early diagnosis

\section{Introduction}

The health of the oral mucosa is directly related to the general health of the human beings. The oral mucosa performs various different functions like protection, sensation and secretion ${ }^{[1]}$. The health of the oral cavity also plays an important in the quality of the life of all individuals [2]. The oral mucous membrane effectively serves as a protective barrier against trauma, pathogens, as well as cancer causing agents ${ }^{[3]}$. Oral mucosal lesions are usually known as any abnormal modification in the color, surface aspects, swelling, or loss of solidarity of the oral mucosal surface. Even though a bigger proportion of OMLs are usually benign and do not require any emergency or active treatment, but there might be cases present demonstrating significant pathology, among these with particular importance are oral potentially malignant disorders which have a greater chance to progress into a malignancy ${ }^{[4]}$. Disruption of the oral health negatively affects a number of important physiologic processes such as speech, chewing and swallowing and it also deteriorates social contacts ${ }^{[5]}$. Oral mucosal lesions are now commonly seen in many populations around the world. The epidemiological studies of oral mucosal lesions are still fewer when compared with studies regarding dental caries or periodontal diseases ${ }^{[6]}$. In India, the consumption of tobacco is responsible for more than half of all the oral mucosal lesions in men and more than one-fourth of oral mucosal lesions in women. The World Health Organization predicts that deaths due to consumption of tobacco in India may exceed 1.5 million annually by 2020 . The incidence of oral mucosal lesions of the oral cavity is maximizing because of the increase in intake of smokeless as well as smoking of tobacco ${ }^{[7]}$. The use of tobacco in India differs from that of the globe. The most widely used form of tobacco globally in the world is in the form of a cigarette, however, in India, merely $20 \%$ of the tobacco consumed is in the form of a cigarette, whereas $40 \%$ is consumed as bidi, and the rest in the form of smokeless/chewable tobacco. 
The use of tobacco in India is influenced by various different factors such as persons attitude, the exposed stress, extensive workload, easy availability, widespread advertising of tobacco brands with different products and a dearth of awareness spreading campaigns ${ }^{[7]}$. There might be many other reasons other than tobacco which may cause oral mucosal lesions such as bacterial, viral or fungal infections, traumatic injuries, systemic diseases and even excessive consumption of betel nut and alcohol ${ }^{[2]}$.

Early diagnosis is the key in the prevention of transformation of oral mucosal lesions into life threatening disease 'Cancer', hence the need of the study determines the prevalence and site distribution of Oral Mucosal Lesions in patients attending outpatient department of Shivam Dental Clinic, Lakhimpur. A single examiner previously trained for the diagnosis of Oral Mucosal lesions made all examinations. The aim of this study was to attain a strategically standard approach based on the guidelines presented by the World Health Organization for the collection and report of data about the oral mucosal lesions.

\section{Materials and Method}

A cross-sectional prevalence study was carried out to assess the prevalence and site distribution of Oral Mucosal Lesions in patients attending outpatient department of Shivam Dental Clinic, Lakhimpur. The patients attending outpatient department of Shivam Dental Clinic, Lakhimpur from 15th of September 2019 to $22^{\text {nd }}$ of February 2020 were included in the study. Patients who were more than 10 years of age and patients who were less than 60 years of age were included in the study. The sample size was calculated using the formula: $\mathrm{N}=\mathrm{Z} 2 \mathrm{P}(1-\mathrm{P}) / \mathrm{D} 2$ and was estimated to be 150 based on the empirical sample size obtained from previous literature. Verbal consent was taken from the patients visiting the outdoor patient department of Shivam Dental Clinic, Lakhimpur. Inclusion Criteria (1). Patients attending outdoor patient department of Shivam Dental Clinic, Lakhimpur. (2) Patients of both genders were included. (3) Patients with age more than 10 years and less than 60 years were included. Exclusion Criteria (1) Patients who were not willing to participate in the study. (2) Patients with systematic disorders. (3) Patients who were less than 10 years and more than 60 years of age. A single examiner previously trained for the diagnosis of Oral Mucosal lesions made all examinations. The examiner was calibrated priorly to ensure uniform interpretation of the data and reliability. Duplicate examinations were conducted for the $5 \%$ of the sample at the beginning, about half way through the survey, and again at the end of the survey to ensure the reliability of the examiner. The data was collected using the WHO oral health assessment form 2013. The oral health surveys provide a concrete basis for assessing the present oral health status of a given population and simultaneously helps to determine the basic needs for oral health care for oral mucosal diseases. The guidelines presented in the WHO oral health assessment form 2013 have been elaborated for practical and economical sample designs suitable for recording the prevalence of oral diseases required for strategic planning and establishment of oral health programmes World Health Organization's Guide to epidemiology and Diagnosis of Oral Mucosal diseases was used as the diagnostic criteria. All patients were examined while seated on a dental chair using artificial light. Two mouth mirrors were used to retract the tongue and the cheeks. Cotton swabs were used during the examination for removing debris and examining whether white lesions could be wiped off or not. The statistical test Pearson's Chi-Square was used to test the significance of the prevalence of the oral mucosal lesions in association with age, gender, and tobacco intake habits. The data analysis was done using the SPSS version 20.

\section{Result}

The present study aimed to attain a strategically standard approach based on the guidelines presented by the World Health Organization for the collection and report of data about the oral mucosal lesions.

A total of 150 study participants were included in the study. Chi square test was applied for independent association of variables.

There was no association between age \& location of lesion $P$ value 0.441

$0.441>0.05=$ non-significant

There was no association between gender \& smoking $P$ value 0.125

$0.125>0.05$ non significance

\section{There was an association between age \& smoking} $P$ value 0.037

$0.037<0.05$ significant

There was an association between age \& chewing tobacco $P$ value 0.000

$0.00<0.05$ significant

\section{There was an association between lesion \& gender}

$P$ value 0.014

$0.014<0.05$ significant

\section{There was an association between age \& lesion \\ $P$ value 0.000 \\ $0.00<0.05$ significant}

There was an association between age $n$ chewing tobacco $P$ value 0.000

$0.000<0.05$ significant

Table 1: Frequency Percent Valid percent

\begin{tabular}{|c|c|c|c|c|}
\hline \multicolumn{2}{|c|}{} & Frequency & Percent & Valid percent \\
\hline \multirow{4}{*}{ Valid } & $20-30$ & 61 & 40.7 & 40.7 \\
\cline { 2 - 5 } & $31-40$ & 47 & 31.3 & 31.3 \\
\cline { 2 - 5 } & $41-50$ & 32 & 21.3 & 21.3 \\
\cline { 2 - 5 } & $51-60$ & 10 & 6.7 & 6.7 \\
\cline { 2 - 5 } & Total & 150 & 100.0 & 100.0 \\
\hline
\end{tabular}

Table 2: Location * age groups cross tabulation count

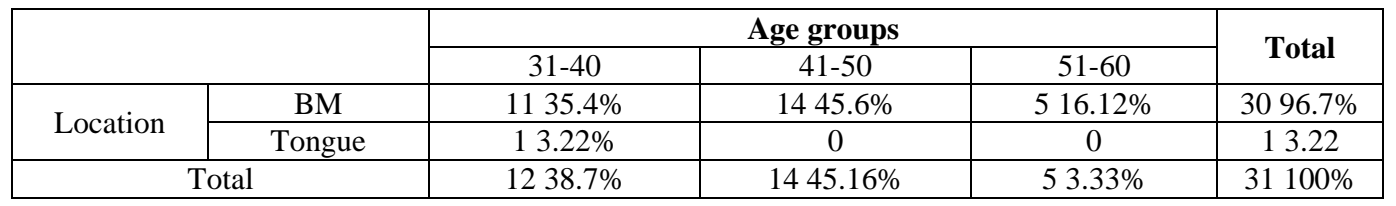


Table 3: Chi-square tests

\begin{tabular}{|c|c|c|c|}
\hline & Value & df & Asymp. sig. (2-sided) \\
\hline Pearson Chi-Square & $1.636^{\mathrm{a}}$ & 2 & .441 \\
\hline Likelihood Ratio & 1.951 & 2 & .377 \\
\hline Linear-by-Linear Association & 1.205 & 1 & .272 \\
\hline N of Valid Cases & 31 & & \\
\hline
\end{tabular}

$P$ value $0.441,0.441>0.05=$ non-significant, There is no association between age $\&$ location of lesion

Table 4: Smoking * gender crosstabulation count

\begin{tabular}{|c|c|c|c|c|}
\hline \multicolumn{2}{|c|}{} & \multicolumn{2}{|c|}{ Gender } & \multirow{2}{*}{ Total } \\
\cline { 3 - 5 } \multicolumn{2}{|c|}{ Smoking } & Male & Female & \\
\hline \multirow{2}{*}{ Non smokers } & $3221.33 \%$ & $117.33 \%$ & $4328.66 \%$ \\
\cline { 2 - 5 } & smokers & $9160.66 \%$ & $1610.66 \%$ & $10771.33 \%$ \\
\hline \multicolumn{2}{|c|}{ Total } & 123 & 27 & 150 \\
\hline
\end{tabular}

Table 5: Chi-square tests

\begin{tabular}{|c|c|c|c|c|c|}
\hline & Value & df & Asymp. sig. (2-sided) & Exact sig. (2-sided) & Exact sig. (1-sided) \\
\hline Pearson Chi-Square & $2.347^{\mathrm{a}}$ & 1 & .125 & & \\
\hline Continuity Correction $^{\mathrm{b}}$ & 1.683 & 1 & .195 & & \\
\hline Likelihood Ratio & 2.230 & 1 & .135 & & \\
\hline Fisher's Exact Test & & & & .158 & .099 \\
\hline Linear-by-Linear Association & 2.332 & 1 & .127 & & \\
\hline N of Valid Cases & 150 & & & & \\
\hline
\end{tabular}

$0.125 p$ value, $0.125>0.05$ no significance, No association between gender \& smoking

Table 6: Crosstab count

\begin{tabular}{|c|c|c|c|c|c|c|}
\hline \multicolumn{2}{|c|}{} & \multicolumn{4}{c|}{ Age groups } & \multirow{2}{*}{ Total } \\
\cline { 3 - 7 } \multicolumn{2}{|c|}{ Smoking } & $\mathbf{2 0 - 3 0}$ & $\mathbf{3 1 - 4 0}$ & $\mathbf{4 1 - 5 0}$ & $\mathbf{5 1 - 6 0}$ & \\
\cline { 2 - 7 } & Non smokers & $2214.66 \%$ & $1610.66 \%$ & $32 \%$ & $21.3 \%$ & $4328.66 \%$ \\
\hline \multicolumn{2}{|c|}{ Smokers } & $396 \%$ & $3120.6 \%$ & $2919.33 \%$ & $85.33 \%$ & $10771.33 \%$ \\
\hline \multicolumn{2}{|c|}{ Total } & $6140.66 \%$ & $4731.3 \%$ & $3221.3 \%$ & $10.66 \%$ & $150100 \%$ \\
\hline
\end{tabular}

Table 7: Chi-square tests

\begin{tabular}{|c|c|c|c|}
\hline & Value & df & Asymp. sig. (2-sided) \\
\hline Pearson Chi-Square & $8.489^{\mathrm{a}}$ & 3 & .037 \\
\hline Likelihood Ratio & 9.775 & 3 & .021 \\
\hline $\begin{array}{c}\text { Linear-by-Linear } \\
\text { Association }\end{array}$ & 5.657 & 1 & .017 \\
\hline N of Valid Cases & 150 & & \\
\hline
\end{tabular}

$0.037<0.05$ significant, Association between age \& smoking

Table 8: Crosstab count

\begin{tabular}{|c|c|c|c|c|}
\hline \multicolumn{2}{|c|}{} & \multicolumn{2}{c|}{ Chewing tobacco } & \multirow{2}{*}{ Total } \\
\cline { 3 - 5 } & Non chewers & Chewers & \\
\hline \multirow{3}{*}{$\begin{array}{c}\text { Age } \\
\text { groups }\end{array}$} & $20-30$ & $2919.33 \%$ & $3221.33 \%$ & $6140.66 \%$ \\
\cline { 2 - 5 } & $31-40$ & $53.33 \%$ & $4228 \%$ & $4731.33 \%$ \\
\cline { 2 - 5 } & $41-50$ & $85.3 \%$ & $2416 \%$ & $3221.33 \%$ \\
\cline { 2 - 5 } & $51-60$ & $53.33 \%$ & $53.33 \%$ & $106.66 \%$ \\
\hline \multicolumn{2}{|c|}{ Total } & $4731.3 \%$ & $10368.66 \%$ & $150100 \%$ \\
\hline
\end{tabular}

Table 9: Chi-square tests

\begin{tabular}{|c|c|c|c|}
\hline & Value & df & Asymp. sig. (2-sided) \\
\hline Pearson Chi-Square & $19.019^{\mathrm{a}}$ & 3 & .000 \\
\hline Likelihood Ratio & 20.399 & 3 & .000 \\
\hline Linear-by-Linear Association & 2.332 & 1 & .127 \\
\hline N of Valid Cases & 150 & & \\
\hline
\end{tabular}

$P$ value $0.000,0.00<0.05$, Association between age \& chewing tobacco

Table 10: Crosstab count

\begin{tabular}{|c|c|c|c|c|}
\hline \multicolumn{2}{|c|}{} & \multicolumn{2}{c|}{ Chewing tobacco } & \multirow{2}{*}{ Total } \\
\cline { 3 - 5 } \multicolumn{2}{|c|}{ Gender } & Non chewers & Chewers & \\
\cline { 2 - 4 } & Male & $3624 \%$ & $8758 \%$ & $12382 \%$ \\
\hline \multicolumn{2}{|c|}{ Total } & $117.3 \%$ & $1610.6 \%$ & $2718 \%$ \\
\hline
\end{tabular}

Table 11: Count

\begin{tabular}{|c|c|c|c|c|}
\hline \multicolumn{2}{|c|}{} & \multicolumn{2}{c|}{ Gender } & \multirow{2}{*}{ Total } \\
\cline { 3 - 4 } \multicolumn{2}{|c|}{ Lesion } & Male & Female & \\
\cline { 2 - 4 } & Present & $9261.33 \%$ & $2617.33 \%$ & $11878.66 \%$ \\
\hline \multicolumn{2}{|c|}{ Total } & $3120.66 \%$ & 10.66 & $3221.33 \%$ \\
\hline
\end{tabular}

Table 12: Chi-square tests

\begin{tabular}{|c|c|c|c|c|c|}
\hline & Value & df & $\begin{array}{c}\text { Asymp. sig. } \\
\text { (2-sided) }\end{array}$ & $\begin{array}{c}\text { Exact sig. } \\
\text { (2-sided) }\end{array}$ & $\begin{array}{c}\text { Exact sig. } \\
\text { (1-sided) }\end{array}$ \\
\hline Pearson Chi Square & $6.098^{\mathrm{a}}$ & 1 & .014 & & \\
\hline Continuity Correction & 4.884 & 1 & .027 & & \\
\hline Likelihood Ratio & 8.067 & 1 & .005 & & \\
\hline Fisher's Exact Test & & & & .010 & .008 \\
\hline $\begin{array}{c}\text { Linear-by-Linear } \\
\text { Association }\end{array}$ & 6.057 & 1 & .014 & & \\
\hline N of Valid Cases & 150 & & & & \\
\hline
\end{tabular}

$P$ value $0.014,0.014<0.05$ significant, Association between lesion \& gender

Table 13: Crosstab count

\begin{tabular}{|c|c|c|c|c|c|}
\hline & \multicolumn{4}{|c|}{ Age groups } & \multirow{2}{*}{ Total } \\
\hline & $21-30$ & $31-40$ & $41-50$ & $51-60$ & \\
\hline Present & $6140.66 \%$ & $3422.66 \%$ & $1812 \%$ & $53.33 \%$ & $11878.6 \%$ \\
\hline Absent & 0 & $138.6 \%$ & $149.3 \%$ & $53.33 \%$ & $3221.33 \%$ \\
\hline Total & $6140.66 \%$ & $4731.3 \%$ & $3221.33 \%$ & $106.6 \%$ & $150100 \%$ \\
\hline
\end{tabular}

Table 14: Chi-square tests

\begin{tabular}{|c|c|c|c|}
\hline & Value & df & Asymp. Sig. (2-sided) \\
\hline Pearson Chi-Square & $32.142^{\mathrm{a}}$ & 3 & .000 \\
\hline Likelihood Ratio & 42.346 & 3 & .000 \\
\hline Linear-by-Linear Association & 30.021 & 1 & .000 \\
\hline N of Valid Cases & 150 & & \\
\hline
\end{tabular}

$P$ value $0.000,0.00<0.05$ significant, Association between age $\&$ lesion 
Table 15: Age groups * Chewingtobacco crosstabulation count

\begin{tabular}{|c|c|c|c|c|}
\hline \multicolumn{2}{|c|}{} & \multicolumn{2}{c|}{ Chewingtobacco } & \multirow{2}{*}{ Total } \\
\cline { 3 - 5 } & $20-30$ & $2919.33 \%$ & $3221.3 \%$ & $6140.66 \%$ \\
\hline \multirow{4}{*}{ Age groups } & $31-40$ & $53.33 \%$ & $4228 \%$ & $4731.33 \%$ \\
\cline { 2 - 4 } & $41-50$ & $85.3 \%$ & $2416 \%$ & $3221.3 \%$ \\
\cline { 2 - 4 } & $51-60$ & $53.33 \%$ & $53.33 \%$ & $106.66 \%$ \\
\hline \multicolumn{2}{|c|}{ Total } & $4731.33 \%$ & $10368.66 \%$ & $150100 \%$ \\
\hline
\end{tabular}

Table 16: Chi-square tests

\begin{tabular}{|c|c|c|c|}
\hline & Value & df & $\begin{array}{c}\text { Asymp. Sig. } \\
\text { (2-sided) }\end{array}$ \\
\hline Pearson Chi-Square & $19.019^{\mathrm{a}}$ & 3 & .000 \\
\hline Likelihood Ratio & 20.399 & 3 & .000 \\
\hline Linear-by-Linear Association & 2.332 & 1 & .127 \\
\hline N of Valid Cases & 150 & & \\
\hline
\end{tabular}
$\mathrm{P}$ value $0.000,0.000<0.05$ significant, Association between age $n$ chewing tobacco Mnb

Table 17: Chewing tobacco

\begin{tabular}{|c|c|c|c|c|c|}
\hline \multicolumn{2}{|c|}{} & Frequency & Percent & $\begin{array}{c}\text { Valid } \\
\text { Percent }\end{array}$ & $\begin{array}{c}\text { Cumulative } \\
\text { Percent }\end{array}$ \\
\hline \multirow{3}{*}{ Valid } & Non cheweers & 47 & 31.3 & 31.3 & 31.3 \\
\cline { 2 - 6 } & chewers & 103 & 68.7 & 68.7 & 100.0 \\
\cline { 2 - 6 } & Total & 150 & 100.0 & 100.0 & \\
\hline
\end{tabular}

Table 18: Smoking

\begin{tabular}{|c|c|c|c|c|c|}
\hline \multicolumn{2}{|c|}{} & Frequency & Percent & $\begin{array}{c}\text { Valid } \\
\text { Percent }\end{array}$ & $\begin{array}{c}\text { Cumulative } \\
\text { Percent }\end{array}$ \\
\hline \multirow{3}{*}{ Valid } & Non smokers & 43 & 28.7 & 28.7 & 28.7 \\
\cline { 2 - 6 } & smokers & 107 & 71.3 & 71.3 & 100.0 \\
\cline { 2 - 6 } & Total & 150 & 100.0 & 100.0 & \\
\hline
\end{tabular}

Table 19: Lesion

\begin{tabular}{|c|c|c|c|c|c|}
\hline \multicolumn{2}{|c|}{} & Frequency & Percent & $\begin{array}{c}\text { Valid } \\
\text { Percent }\end{array}$ & $\begin{array}{c}\text { Cumulative } \\
\text { Percent }\end{array}$ \\
\hline \multirow{3}{*}{ Valid } & Absent & 118 & 78.7 & 78.7 & 78.7 \\
\cline { 2 - 6 } & Present & 32 & 21.3 & 21.3 & 100.0 \\
\cline { 2 - 6 } & Total & 150 & 100.0 & 100.0 & \\
\hline
\end{tabular}

Table 20: Carcinoma

\begin{tabular}{|c|c|c|c|c|c|}
\hline \multicolumn{2}{|c|}{} & Frequency & Percent & $\begin{array}{c}\text { Valid } \\
\text { Percent }\end{array}$ & $\begin{array}{c}\text { Cumulative } \\
\text { Percent }\end{array}$ \\
\hline \multirow{3}{*}{ Valid } & Absent & 149 & 99.3 & 99.3 & 99.3 \\
\cline { 2 - 6 } & Present & 1 & .7 & .7 & 100.0 \\
\cline { 2 - 6 } & Total & 150 & 100.0 & 100.0 & \\
\hline
\end{tabular}

Table 21: Leukoplakia

\begin{tabular}{|c|c|c|c|c|c|}
\hline \multicolumn{2}{|c|}{} & Frequency & Percent & $\begin{array}{c}\text { Valid } \\
\text { percent }\end{array}$ & $\begin{array}{c}\text { Cumulative } \\
\text { percent }\end{array}$ \\
\hline \multirow{3}{*}{ Valid } & Absent & 141 & 94.0 & 94.0 & 94.0 \\
\cline { 2 - 6 } & Present & 9 & 6.0 & 6.0 & 100.0 \\
\cline { 2 - 6 } & Total & 150 & 100.0 & 100.0 & \\
\hline
\end{tabular}

Table 21: Erythroplakia

\begin{tabular}{|c|c|c|c|c|c|}
\hline \multicolumn{2}{|l|}{} & Frequency & Percent & $\begin{array}{c}\text { Valid } \\
\text { Percent }\end{array}$ & $\begin{array}{c}\text { Cumulative } \\
\text { Percent }\end{array}$ \\
\hline Valid & absent & 150 & 100.0 & 100.0 & 100.0 \\
\hline
\end{tabular}

Table 22: Lichen planus

\begin{tabular}{|c|c|c|c|c|c|}
\hline \multicolumn{2}{|c|}{} & Frequency & Percent & $\begin{array}{c}\text { Valid } \\
\text { Percent }\end{array}$ & $\begin{array}{c}\text { Cumulative } \\
\text { Percent }\end{array}$ \\
\hline \multirow{3}{*}{ Valid } & Absent & 137 & 91.3 & 91.3 & 91.3 \\
\cline { 2 - 6 } & Present & 13 & 8.7 & 8.7 & 100.0 \\
\cline { 2 - 6 } & Total & 150 & 100.0 & 100.0 & \\
\hline
\end{tabular}

Table 23: OSMF

\begin{tabular}{|c|c|c|c|c|c|}
\hline \multicolumn{2}{|c|}{} & Frequency & Percent & $\begin{array}{c}\text { Valid } \\
\text { Percent }\end{array}$ & $\begin{array}{c}\text { Cumulative } \\
\text { Percent }\end{array}$ \\
\hline \multirow{3}{*}{ Valid } & Absent & 142 & 94.7 & 94.7 & 94.7 \\
\cline { 2 - 6 } & Present & 8 & 5.3 & 5.3 & 100.0 \\
\cline { 2 - 6 } & Total & 150 & 100.0 & 100.0 & \\
\hline
\end{tabular}

Table 24: Candidiasis

\begin{tabular}{|c|c|c|c|c|c|}
\hline \multicolumn{2}{|c|}{} & Frequency & Percent & $\begin{array}{c}\text { Valid } \\
\text { Percent }\end{array}$ & $\begin{array}{c}\text { Cumulative } \\
\text { Percent }\end{array}$ \\
\hline \multirow{3}{*}{ Valid } & Absent & 149 & 99.3 & 99.3 & 99.3 \\
\cline { 2 - 6 } & Present & 1 & .7 & .7 & 100.0 \\
\cline { 2 - 6 } & Total & 150 & 100.0 & 100.0 & \\
\hline
\end{tabular}

Table 25: Gender

\begin{tabular}{|c|c|c|c|c|c|}
\hline \multicolumn{2}{|c|}{} & Frequency & Percent & $\begin{array}{c}\text { Valid } \\
\text { Percent }\end{array}$ & $\begin{array}{c}\text { Cumulative } \\
\text { Percent }\end{array}$ \\
\hline \multirow{3}{*}{ Valid } & Male & 123 & 82.0 & 82.0 & 82.0 \\
\cline { 2 - 6 } & Female & 27 & 18.0 & 18.0 & 100.0 \\
\cline { 2 - 6 } & Total & 150 & 100.0 & 100.0 & \\
\hline
\end{tabular}

\section{Discussion}

Early diagnosis is the key in the prevention of transformation of oral mucosal lesions into life threatening disease 'CANCER', hence the need of the study determines the prevalence and site distribution of Oral Mucosal Lesions in patients attending outpatient department of Shivam Dental Clinic, Lakhimpur.

$78 \%$ of the study participants had an oral mucosal lesion which was contrasting according to a study by Andrej Aleksander Kansky et al, Shakir Mahmood Al-Gburi et al. and Sendhil Kumar et al. ${ }^{[2,5,6]}$.

In the current study the age group most affected by oral mucosal lesions was 21-30 years (41\%) where as in a study conducted by Kamla A et al. ${ }^{[3]}$ and Kaveri Hallikeri et al. it was more than 40 years ${ }^{[7]}$.

The most common oral mucosal lesion in the present study was oral lichen planus $(8.7 \%)$ while it was hairy tongue (17.4\%), cheek biting and fordyces granules in studies conducted by Sami El Toum et al. ${ }^{[4]}$, Aleksander Kansky et al. ${ }^{[5]}$ and Daud Mirza et al. ${ }^{[11]}$.

The most common affected site by the oral mucosal lesions was the buccal mucosa $(40.5 \%)$ followed by dorsum of the tongue $(27 \%)$ in a study conducted by Maher Alshayeb et al. ${ }^{[8]}$ which was also in accordance with the current study where the most common site for oral mucosal lesions was buccal mucosal followed by the tongue.

There was no significant difference in the incidence of oral lesions between the genders in the present study and was comparable with a study conducted by Meral ÜNÜR et al. and Cury, Patricia Ramos et al. ${ }^{[9,10]}$.

In the current study minimum number of oral mucosal lesions were found in the age group above 50 years of age while the least affected age group was 10-20 years in a study conducted by Daud Mirza et al. ${ }^{[11]}$.

In the current study the female participants were $18 \%$ which was more than in a study conducted by M Krishna Priya et al. ${ }^{[12]}$ and less than studies conducted by K. M. Shivakumar et al. ${ }^{[13]}$ and Ali-Rıza-İlker Cebeci et al. ${ }^{[14]}$.

The total number of study participants in the present study were 150 where as in studies conducted by José Nicolau Gheno et al. ${ }^{[15]}$, Arvind Babu et al. ${ }^{[16]}$ and Prashant N. Keche et al. ${ }^{[17]}$. were 801,3500 and 255 respectively. 


\section{Conclusion}

Oral mucosal lesions have a potential tendency to transform in oral mucosal malignancies which ultimately result in death. The present study concludes a positive relation between intake of tobacco in any form with oral mucosal lesions hence an additional effort to educate the masses about the hazardous effects of tobacco should be a priority for both governmental and non-governmental agencies.

\section{Conflicts of Interest: None}

\section{References}

1. Yadav N, Jain M, Sharma A, Yadav R, Pahuja M, Jain V. Distribution and prevalence of oral mucosal lesions in residents of old age homes in Delhi, India. NJE 2018;8(2):727-734.

2. Shakir Mahmood Al-Gburi, Shaimaa Hamid Mudhir, The prevalence of the oral mucosal lesions among adult patients in Abu Ghraib city Iraq. J Res Med Dent Sci 2018;6(5):145-148.

3. Kamble KA, Guddad SS, Nayak AG, SuragimathA, Sanade AR. Prevalence of Oral Mucosal Lesions in Western Maharashtra A Prospective Study. J Indian Acad Oral Med Radioly 2017;29(4):282-287.

4. Toum S, Cassia A, Bouchi N, Kassab I. Prevalence and Distribution of Oral Mucosal Lesions by Sex and Age Categories: A Retrospective Study of Patients Attending Lebanese School of Dentistry. International Journal of Dentistry 2018;27(8):1-6.

5. Aleksander Kansky A, Didanovic V, Dovsak T, Loncar B, Pelivan I, Terlevic D. Epidemiology of oral mucosal lesions in Slovenia. Radiol Oncol 2018;52(3):263-266.

6. Kumar S, Narayanan VS, Ananda S R, Kavitha A P, Krupashankar R. Prevalence and risk indicators of oral mucosal lesions in adult population visiting primary health centers and community health centers in Kodagu district. J Family Med Prim Care 2019;8(7):2337-42.

7. Hallikeri K, Naikmasur V, Guttal K, Shodan M, Chennappa NK. Prevalence of oral mucosal lesions among smokeless tobacco usage: A cross-sectional study. Indian J Cancer 2018;55(6):404-9.

8. Alshayeb M, Mathew A, Varma S, Elkaseh A, Kuduruthullah S, Ashekhi A, Wahab A. Habbal, Prevalence and distribution of oral mucosal lesions associated with tobacco use in patients visiting a dental school in Ajman. Oncology and Radiotherapy 2019;46(1):029-033.

9. Ünür M, Bektaş-Kayhan K, Seda Altop M, Boy-Metin Z, Keskin Y. The prevalence of oral mucosal lesions in children A single center study. J Istanbul Univ Fac Dent 2015;49(3):29-38.

10. Cury Ramos P, Arruda L, Santos D, Nunes J, Ribeiro E, Figueiredo L, Xavier D, Caló F, Figueiredo, Leal A, Ramalho, Pedreira L. Prevalence and risk indicators of oral mucosal lesions in adult population visiting primary health centers and community health centers in Kodagu district. Journal of Medicine 2014;93(27):140-153.

11. Mirza D, Karim Z, Marath M, Ahmed A, Zaidi N. Frequency and distribution of oral mucosal lesions: a cross-sectional study. PODJ 2017;37(1):45-48.

12. Krishna Priya M, Srinivas P, Devaki T. Evaluation of the prevalence of oral mucosal lesions in a population of eastern coast of South India. J Int Soc Prevent Communit Dent 2018;8:396-401.

13. Shivakumar K, Kadashetti V, Chaudhary M, Patil S,
Gawande M, Hande A, et al. Prevalence of Oral Mucosal Lesions in Patients with Dermatological Diseases Attending Tertiary Care Hospital in Central India. JKIMSU 2017;6(3):55-61.

14. Cebeci A, Gülşahı A, Kamburoğlu K, Orhan B, Öztaş B. Prevalence and distribution of oral mucosal lesions in an adult turkish population. Journal section: Oral Medicine and Pathology 2009;14(6):272-277.

15. Gheno J, Martins M, Munerato M, Hugo F, Filho M, Weissheimer C, et al, Martins M. Oral mucosal lesions and their association with sociodemographic, behavioral, and health status factors. Brazil oral res 2015;29(1):1-19.

16. Arvind Babu RS, Chandrashekar P, Kumar KK, Reddy GS, Prakash Chandra KL Rao. A study on oral mucosal lesions in 3500 patients with dermatological diseases in South India. Ann Med Health Sci Res 2014;4(2):84-93

17. Keche PN, Gadpayle NP, Gawarle SH, Chamania GA. An observational study of benign oral lesions in central India. Int $\mathbf{J}$ Otorhinolaryngol Head Neck Surg 2017;3(4):816-20. 